\title{
Norois
}

Environnement, aménagement, société

$233 \mid 2014$

Mobilité, santé et développement territorial : de nouveaux défis pour la gouvernance des territoires ruraux

\section{Du potentiel à l'action : la gouvernance territoriale des pôles d'excellence rurale}

Building-up collective action: place-based governance in the "rural excellence poles"

Sylvie Lardon, Johan Milian, Salma Loudiyi, Patrice LeBlanc, Laurence Barthe et François Taulelle

\section{(2) OpenEdition}

Édition électronique

URL : https://journals.openedition.org/norois/5380

DOI : 10.4000/norois. 5380

ISBN : 978-2-7535-4127-6

ISSN : $1760-8546$

Éditeur

Presses universitaires de Rennes

Édition imprimée

Date de publication : 20 décembre 2014

Pagination : 69-81

ISBN : 978-2-7535-4083-5

ISSN : 0029-182X

\section{Référence électronique}

Sylvie Lardon, Johan Milian, Salma Loudiyi, Patrice LeBlanc, Laurence Barthe et François Taulelle, « Du potentiel à l'action : la gouvernance territoriale des pôles d'excellence rurale », Norois [En ligne], 233 |

2014, mis en ligne le 20 décembre 2016, consulté le 14 janvier 2022. URL : http://

journals.openedition.org/norois/5380; DOI : https://doi.org/10.4000/norois.5380 


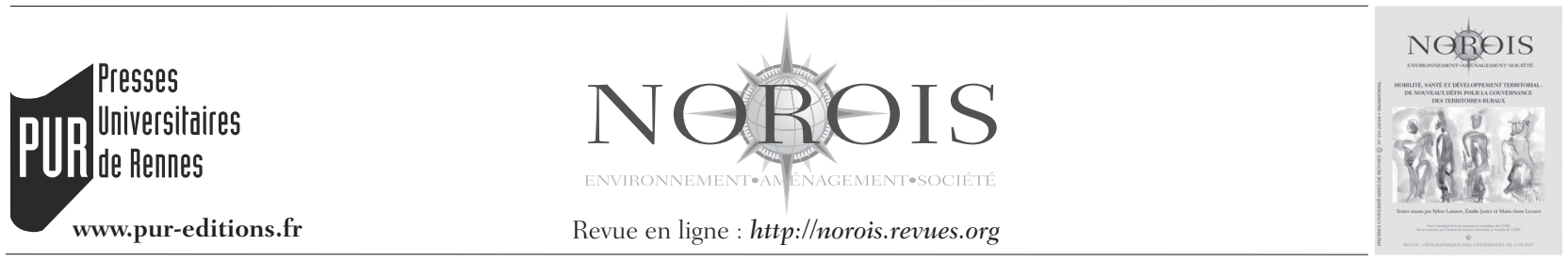

\title{
Du potentiel à l'action : la gouvernance territoriale des pôles d'excellence rurale
}

\author{
Building-up Collective Action: Place-based Governance in the "Rural Excellence Poles"
}

\author{
Sylvie LaRdon ${ }^{* a}$, Johan Milian ${ }^{b}$, Salma LoudiYic, \\ Patrice LeBlanc ${ }^{\mathrm{d}}$, Laurence BARTHE ${ }^{\mathrm{e}}$, François TAULELle ${ }^{\mathrm{f}}$
}

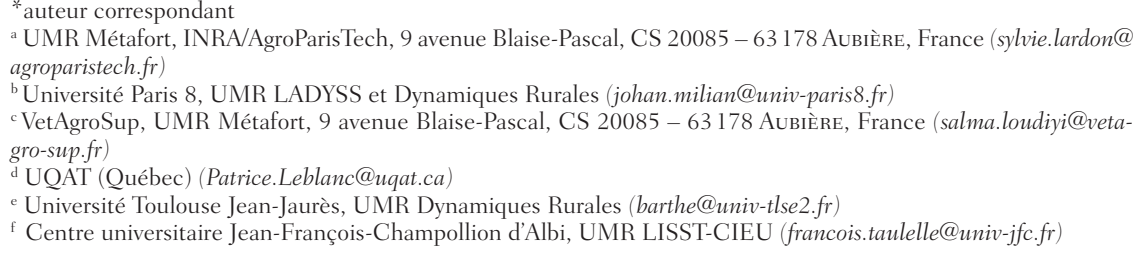

Résumé : Cet article analyse les effets des pôles d'excellence rurale (PER) en matière de construction de la gouvernance territoriale. Il s'appuie sur deux recherches appliquées à des territoires porteurs de PER. Pour caractériser les dynamiques sociales et spatiales constitutives de ces territoires, la première recherche a travaillé sur les dossiers de candidature à la labellisation PER au moyen d'une grille des configurations socio-spatiales. Sur un corpus de PER en partie différent, la seconde recherche a interrogé le panel des acteurs parties-prenantes impliqués dans l'élaboration et l'animation des PER, afin de reconstituer leur élaboration et d'étudier leur ancrage dans les trajectoires de développement de ces territoires. Le croisement de ces deux approches sur quelques PER communs met en évidence les leviers d'actions possibles pour le développement des territoires. Les configurations socio-spatiales donnent à voir le potentiel de développement des projets PER et les trajectoires de développement valident ou non la mise en œeuvre de la gouvernance territoriale. Elles sont ainsi appréhendées à la fois comme des révélateurs des processus de développement et comme des activateurs de nouveaux modèles pour les territoires ruraux.

Abstract: This article analyses the effects of Rural Excellence Poles (PER) on the development of territorial governance. PER have been advocated by the French Ministry of Regional Planning as a key element for the promotion and the organisation of rural development. PER framework was a financial tool that supported 670 local initiatives. This paper focuses on two research studies based on territories implementing a PER. To characterise the social and spatial dynamics of these spaces, the first study analysed PER application files using a socio-spatial pattern grid. Based on a similar corpus, the second study interviewed a panel of stakeholders involved in the making and the animation of PER to reconstruct their elaboration and study how they are anchored in the development trajectories of these territories. Socio-spatial configurations highlight the development potential of PER projects while development trajectories determine if territorial governance benefits from PER. Socio-spatial configurations are acknowledged as indicators of development processes and as activators of new models for rural territories.

Mots clés : système d'acteurs - action collective - projet de territoire - configuration socio-spatiale - trajectoire de développement

Keywords: local system stakeholders - collective action - territorial project - socio-spatial configuration - development trajectories 


\section{INTRODUCTION}

Les territoires ruraux sont confrontés à des opportunités et des menaces qui, à un moment ou à un autre, guident leurs choix de développement et convoquent l'intelligence collective des acteurs (Jean et Vanier, 2008). Dans le cadre de la territorialisation des politiques publiques, divers acteurs sont amenés à initier des actions localisées et à les mettre en œuvre dans des projets de territoire. Ces projets de territoire contribuent au développement territorial, défini comme «l'augmentation de la capacité des acteurs à maîtriser les dynamiques d'évolution qui les concernent» (Deffontaines et al., 2001). Ils relèvent d'une gouvernance territoriale, définie comme un "processus d'articulation dynamique de l'ensemble des pratiques et des dispositifs institutionnels entre des acteurs géographiquement proches en vue de résoudre un problème productif ou de réaliser un projet de développement » (Gilly et al., 2004). L'étude de ce processus s'intéresse donc à la construction des arbitrages et des coordinations entre les différents acteurs impliqués dans le débat territorial, qu'ils soient institutionnels, politiques, économiques ou sociaux (Pasquier et al., 2007).

Les modalités de gouvernance territoriale peuvent prendre des formes diverses. Elles se construisent à l'aide de compétences en matière d'ingénierie territoriale et d'accompagnement de l'action (Lardon et al., 2008). Nous abordons ici la procédure de labellisation des pôles d'excellence rurale (PER) pour apprécier les évolutions et recompositions dans les jeux d'acteurs dont elle témoigne, et rendre compte du territoire comme un système dynamique organisé. Portée par un appel à projets, cette labellisation des PER donne ainsi accès à une grande diversité de projets proposés et présentés par les territoires eux-mêmes. Leur étude fournit un large panorama de la genèse des actions que mènent des acteurs locaux face aux enjeux de développement, des choix qu'ils privilégient et des modes de coordination et de décision qui s'opèrent dans les territoires.

L'article est structuré en trois parties. La première partie présente les deux approches mobilisées dans nos travaux pour étudier les mécanismes de la gouvernance des territoires ruraux au prisme des PER. La première approche élabore une grille des configurations socio-spatiales caractérisant les dynamiques sociales et spatiales des territoires de projet
(Lardon et Cayre, 2009). La seconde approche identifie quelles sont les formes de plus-value que les PER génèrent par l'analyse de leur inscription dans les trajectoires de développement des territoires (Barthe, 2009). La deuxième partie montre que les PER étudiés révèlent un potentiel de développement qui s'exprime par une gouvernance effective sur le terrain, si tant est que les actions prévues sont réalisées. Bien que les résultats soient mitigés, l'analyse met en évidence des leviers d'actions possibles pour le développement des territoires. La troisième partie propose quelques pistes, tant pour accompagner les dynamiques d'acteurs par une nouvelle ingénierie territoriale que pour élaborer une politique de développement territorial en lui donnant un cadre institutionnel. Cela ouvre des perspectives de recherche nouvelles, à l'heure où les recompositions territoriales sont en débat.

\section{Caractériser la gouvernance TERritoriale dANS LES PER}

La DATAR a engagé fin 2005 un appel à projets auprès des territoires ruraux pour constituer des Pôles d'Excellence Rurale (PER) ${ }^{1}$. Cette démarche de labellisation avait pour objectif de soutenir financièrement des investissements censés contribuer au développement des territoires ruraux. La DATAR a par ailleurs initié en 2008 un programme de recherche évaluative ${ }^{2}$ pour assurer un suivi qualitatif de ces PER et alimenter la réflexion des pouvoirs publics à l'égard des territoires ruraux. Les travaux présentés ici ont été conduits dans ce cadre par deux équipes de recherche rattachées respectivement à l'UMR Métafort de Clermont-Ferrand et à l'Université Jean-Jaurès de Toulouse.

Nos équipes ont ainsi chacune étudié un corpus de PER spécifiques issus de la première génération (2007-2010), en suivant des approches complémentaires. L'une met en avant les configurations sociospatiales à partir de l'analyse des documents de projet, l'autre relève les trajectoires de développement à partir d'entretiens avec les porteurs de projet.

\footnotetext{
1. [http://poles-excellence-rurale.datar.gouv.fr/].

2. [http://www.datar.gouv.fr/programme-de-recherche-evaluative-sur-les-polesdexcellence-rurale-2009].
} 


\section{Les pôles d'excellence rurale dans le paysage des dispositifs de développement rural}

Un PER est un projet de développement économique $^{3}$ généralement piloté par une collectivité, une intercommunalité ou un établissement de coopération, portant sur la réalisation d'investissements matériels censés jouer un rôle d'équipements structurants pour des activités de services ou de production. Le dispositif PER soutenait donc théoriquement des initiatives locales. Thématiquement les PER devaient concerner des secteurs d'activité identifiés par la DATAR comme spécifiques des fonctions qu'elle attribuait alors aux espaces ruraux, dans le champ de l'économie productive ou celui de l'économie résidentielle. Quatre secteurs étaient plus particulièrement ciblés : tourisme, bio-ressources, services et petites industries locales. La réalisation de ces projets devait être rapide, sous trois ans une fois la labellisation obtenue, afin notamment de donner une forte visibilité au dispositif. Celui-ci a été reconduit en 2009 dans le cadre d'un second appel à projets positionné sur les mêmes thématiques avec une attention plus marquée sur les projets en lien avec les services. La DATAR a finalement labellisé 670 PER sur les deux générations.

Les PER s'inscrivent dans la philosophie des théories relatives aux pôles de croissance émises dans les années 1950 par des économistes comme François Perroux. Dans le contexte politique de 2005, la promotion du modèle du "pôle » dans le champ du développement rural prétend alors rompre avec une logique de « rétribution » incarnée par les politiques de zonages privilégiant une logique d'assistance (Taulelle, 2008) au profit d'une logique distinctive fondée sur la figure du « projet structurant » et « la capacité des territoires à savoir s'organiser » (Guigou, 2008). Le dispositif promeut donc une action volontariste, centrée sur les investissements et orientée vers une vision libérale et entrepreneuriale du développement qui serait en mesure de «mobiliser les potentiels de compétitivité » pas ou insuffisamment exploités et d'investir des domaines

3. Les PER ont été une sorte de compensation à la politique des pôles de compétitivité engagée en faveur des métropoles et des systèmes industriels. L'Etat critiqué par les associations des maires des villes petites et moyennes et des élus locaux de l'espace rural s'est vu contraint de proposer une initiative pour les territoires ruraux (il excluait les aires urbaines de plus de 30000 habitants). Cependant les sommes mobilisées étaient sans commune mesure avec les financements des pôles de compétitivité. économiques «hors des sentiers battus » (Alvergne et De Roo, 2008). Cette vision performative du dispositif confère aux PER alors en puissance une capacité de territorialisation endogène, le PER constitué étant censé venir nourrir une systémogenèse de développement territorial (Woessner, 2010; Milian et Bacconnier, 2014).

Dans cette rhétorique, des effets étaient attendus en matière de « gouvernance de projet et de collaboration entre acteurs » puisqu'il s'agissait d'un des cinq critères d'évaluation des candidatures. Les PER devaient constituer des scènes de discussion de projet et permettre de dégager des périmètres de coopération. En mettant notamment en exergue la question des partenariats publics-privés et du soutien aux initiatives des petits porteurs de projet, le dispositif espérait encourager des pratiques de gouvernance territoriale mixte où « les acteurs qui pilotent la coordination locale sont des acteurs publics et aussi des privés qui travaillent ensemble sur un projet commun de développement » (Leroux, 2006).

\section{L'approche par les configurations socio-spatiales}

L'équipe clermontoise s'est centrée sur l'élaboration d'une grille d'analyse des configurations sociospatiales des PER, à partir des dossiers de réponse à l'appel à projets PER (Lardon et al., 2012). Cette grille d'analyse vise à décrypter les dynamiques territoriales associées à la mise en ouvre d'un PER et à identifier comment ces derniers peuvent être un levier d'action dans les territoires. Elle spécifie les dynamiques de relations sociales nécessaires à la construction de l'action collective (Angeon et al., 2006). Elle explicite leur ancrage territorial et les modèles spatiaux qui résultent des dynamiques territoriales (Lardon et Piveteau, 2005). À cet égard, il s'agit d'un repérage des potentiels de développement, des ingrédients nécessaires à la valorisation des ressources territoriales par les acteurs (Gumuchian et Pecqueur, 2007).

La méthodologie s'appuie sur l'analyse des dossiers PER; elle a été élaborée sur les 21 dossiers de la région Auvergne (Perraud, 2009). Les configurations socio-spatiales sont caractérisées par la répartition spatiale, concentrée ou diffuse, des acteurs et des actions dans le territoire de projet et les liens, établis ou non, avec des acteurs extérieurs. Elles sont croi- 
sées avec la façon dont les différents types d'acteurs (institutionnels, consulaires, associatifs, entreprises, recherche) s'impliquent dans la construction du projet PER. Les modèles ainsi élaborés donnent à voir la contribution des actions au projet de territoire (figure 1).
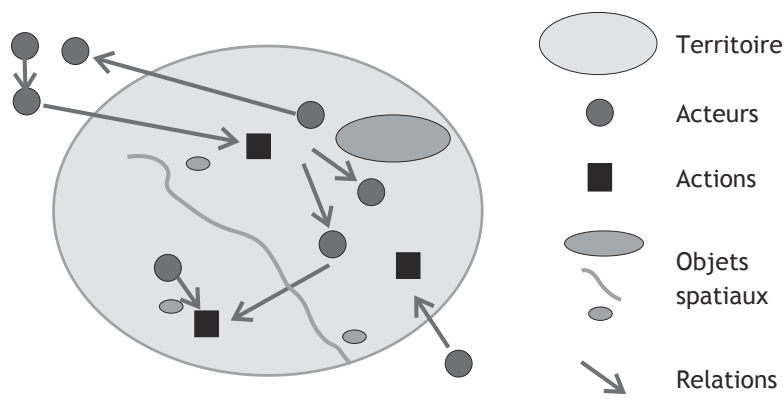

Figure 1 : Configuration socio-spatiale Socio-spatial configuration

L'approche par les configurations socio-spatiales a permis une première caractérisation du potentiel de développement porté par le projet PER, à partir des dossiers de candidature. Le potentiel et les capacités de développement d'un territoire de projet sont caractérisés par l'enchaînement logique des événements qui contribuent à la genèse du projet. En particulier, il est important de spécifier à quel moment intervient l'institution porteuse du projet, si elle est plutôt initiatrice, fédératrice ou porteuse de la dynamique et à quel moment interviennent d'autres types d'acteurs (par exemple des entreprises, des associations, le monde de l'enseignement-recherche, l'expertise). La reconstruction de la genèse du projet PER s'organise selon un axe de développement révélateur des synergies opérées entre les acteurs et des marges de manœuvre pour réaliser leur projet.

Le potentiel de développement identifié à partir des dossiers PER selon la méthodologie précédemment établie a été confronté à la réalité terrain par des enquêtes menées auprès des porteurs de projets (Ider, 2010). Cette analyse a été faite sur un échantillon de 25 PER qu'étudiaient par ailleurs d'autres équipes du groupe de recherche évaluative dans le Sud-Ouest (Barthe et al., 2009), le Massif central (Mainet et al., 2009) ou la région Rhône-Alpes (Pecqueur et al., 2009) ou par l'équipe Clermontoise (figure 2). La typologie des actions a été affinée, en distinguant les actions de construction, d'aménagement et de gestion et les relations de financement, de conseil et d'animation et les liens de travail et de voisinage, permettant de mieux comprendre le rôle des acteurs dans la conduite du projet. La confrontation terrain sur les quelques cas de la région Auvergne a permis de valider l'identification du potentiel de développement à partir de l'analyse des dossiers PER, selon la méthodologie des configurations socio-spatiales. Le taux de réalisation des actions (écart entre ce qui est indiqué dans le dossier et ce qui est mis en œuvre sur le terrain) a également pu être évalué.

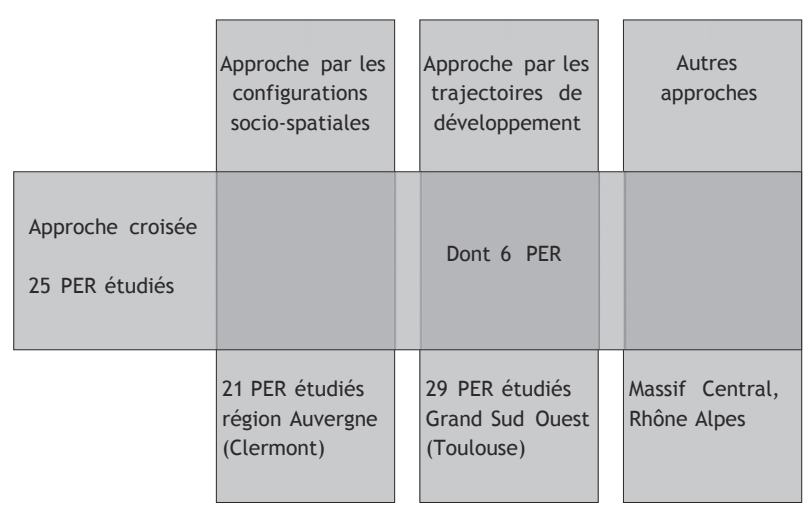

Figure 2 : Corpus des PER étudiés et croisés Presentation of REP sample surveyed

\section{L'approche par les trajectoires de développement}

L'équipe de l'Université de Toulouse a conduit une enquête approfondie sur un échantillon de 29 PER du Sud-Ouest de la France en vue de caractériser leur place et leur rôle dans les trajectoires de développement des territoires porteurs de la candidature. Les dynamiques et les changements qui marquent les trajectoires (Berger et al., 2010; Milian et Bacconnier, 2014) peuvent s'interpréter à travers les continuités, les inflexions, voire les ruptures qui s'opèrent dans la (re)définition des ressources sur lesquelles un système territorial appuie son développement (activation et valorisation de ces ressources) ainsi que les recompositions organisationnelles et structurelles dont il se dote en ce sens. Ces recompositions ont pu concerner par exemple la création d'établissements de coopération, voire 
des structures associant des acteurs publics et privés (types SEM, SCIC, GIE) pour réagencer une filière économique ou bien répondre à des besoins nouveaux pour la population. L'objectif était de retracer l'évolution de la capacité d'initiative du système local et de reconstituer la manière dont il s'était investi dans des démarches «innovantes » (variables en fonction des périodes : par exemple la conception de SCoTs en zone rurale, le lancement d'une démarche de type Plan Climat-EnergieClimat-Territoire etc.) et s'approprier la méthodologie du projet de territoire. Ce second aspect dans la caractérisation des trajectoires permettait d'étudier finement si le PER contribuait à la construction ou la consolidation d'une gouvernance territoriale, puisqu'il amenait à étudier le système d'animation et de décision à l'œuvre autour de la trajectoire de développement du territoire et les innovations et recompositions dont il était porteur ou catalyseur.

Cette recherche a étudié finement les contextes de montage et de réalisation des PER, en appréciant comment le dispositif participait à la redéfinition des ressources territoriales, et, le cas échéant, venait infléchir les stratégies des acteurs institutionnels comme socioprofessionnels et faire évoluer les systèmes d'action. L'étude a ainsi abordé les modalités d'émergence du PER, les méthodes d'animation et de sélection des actions, ainsi que les impacts de ces actions sur le système d'acteurs. L'analyse s'est appuyée sur un corpus d'une centaine d'entretiens semi-directifs auprès de trois catégories d'acteurs : des animateurs et accompagnateurs du dispositif (services déconcentrés de l'État, collectivités territoriales, c'est-à-dire les partenaires financiers publics), des concepteurs locaux des PER, notamment les décideurs et opérateurs locaux (élus, personnels des collectivités, ingénierie privée d'appui, co-financeurs privés), enfin des bénéficiaires des aides (organismes publics, associations, particuliers et entreprises). La participation à des réunions de travail (programmation des financements, comités de pilotage) dans certains PER a complété les enquêtes.

\section{LA GOUVERNANCE DES PÔLES D'EXCELLENCE RURALE}

Les deux approches ont été menées sur des corpus en partie différents, mais elles ont pu être croisées sur quelques cas. Les résultats s'avèrent complé- mentaires pour aborder la question de la gouvernance des PER et convergents pour rendre compte des atouts et limites du dispositif à cet égard.

Chacune des approches a mené à la constitution d'une typologie, basée sur le croisement entre réalisation des actions et dynamiques de développement pour l'équipe clermontoise et sur le croisement entre gouvernance territoriale et réalisation des actions pour l'équipe toulousaine. Les 6 PER communs aux deux études ont servi à croiser les approches et à évaluer le lien entre potentiel de gouvernance territoriale et mise en œuvre effective (Lardon et al., 2014). L'ensemble des PER analysés a fourni des éléments de réflexion sur ce qu'a été la gouvernance des territoires PER.

\section{Du potentiel de gouvernance territoriale...}

Ainsi, sur le corpus étudié des 21 PER de la région Auvergne, nous avons mis en évidence des effets différenciés de gouvernance territoriale. Les formes de PER portées par des acteurs institutionnels ont eu peu d'effet, car elles n'ont souvent fait que reproduire des situations existantes, où la place des acteurs était déjà fixée. A contrario, les initiatives d'entreprises ou d'associations, relayées et légitimées ensuite par l'acteur institutionnel, se sont révélées plus porteuses de dynamiques, car elles innovaient tant dans la forme que dans les relations nouvelles entre acteurs, en particulier par l'hybridation publicprivé (aussi bien pour des projets touristiques foisonnants que pour des services plus ciblés comme les maisons médicales). Le rôle intermédiaire des acteurs de la formation et de la recherche a souvent catalysé la mise en synergie pour des actions collectives coordonnées (en particulier dans les projets sur le bois-énergie).

La dynamique de développement engendrée par les PER, identifiée à partir des dossiers (Perraud, 2009) et vérifiée par enquête (Ider, 2010), nous amène à distinguer 5 types de PER (figure 3). Un PER « défectueux » n'a pas réalisé ses objectifs initiaux en termes de réalisation des actions, même s'il a pu apporter un plus aux institutions porteuses, par exemple en termes de légitimité, comme ça peut être le cas pour des PER portés par des communautés de communes, qui n'ont souvent pas assez d'envergure, mais qui sont montées en capacité avec cette expérience. Un 


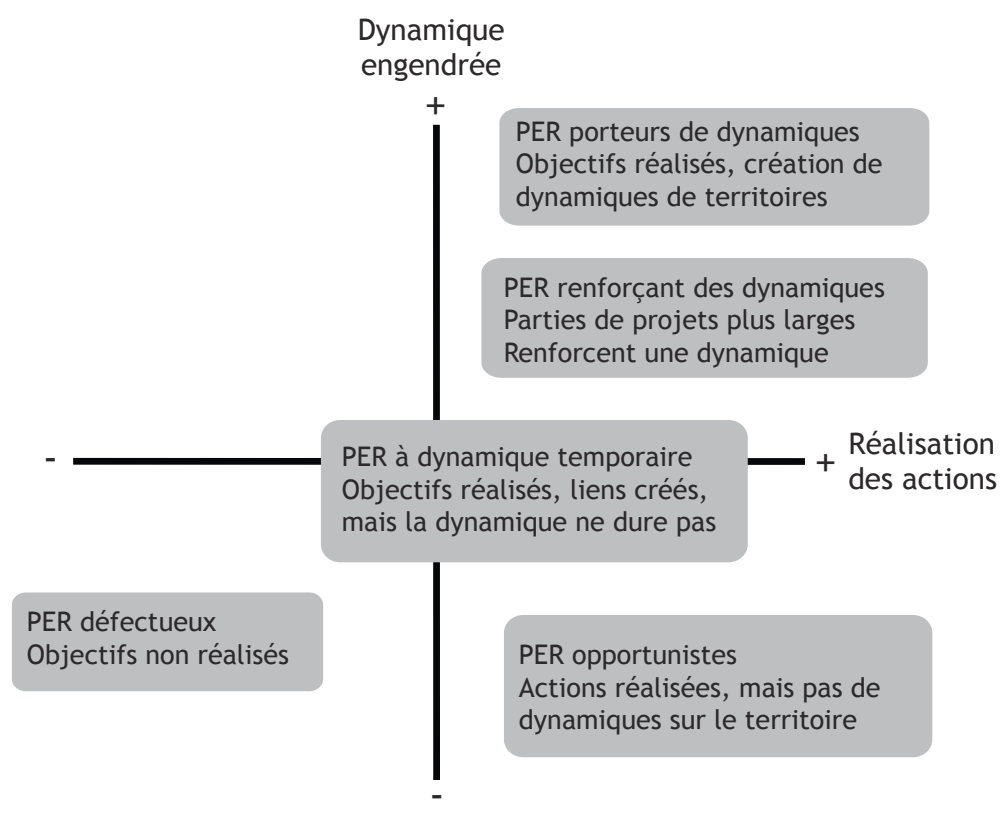

Figure 3 : Typologie de la dynamique territoriale engendrée et de la réalisation des actions, par l'approche des configurations socio-spatiales (d'après Lardon et al., 2014)

A typology of territorial dynamics and achievements using socio-spatial configuration methodology
$\mathrm{PER}$ « opportuniste » a réalisé ses actions, mais sans engendrer de dynamique territoriale nouvelle, notamment lorsque les projets sont liés à la valorisation de produits agricoles ou touristiques sans que cela n’ait de répercussion notable sur les autres activités du tissu socio-économique du territoire. Un PER à « dynamique temporaire » a pu réaliser ses objectifs de départ en termes d'actions, mais sans que les liens créés n'entrainent une dynamique pérenne, souvent par essoufflement du collectif d'acteurs et manque de relais dans l'animation et la conduite du projet. Un PER qui « renforce une dynamique » correspond à des actions abouties qui s'inscrivent dans un projet plus large et viennent renforcer une démarche préexistante sur le territoire, ce qui est le cas soit dans des démarches institutionnelles engagées de longue date ou dans des territoires où le projet est déjà bien ancré. Il s'appuie sur une dynamique déjà existante pour la renforcer et la relayer. Enfin, un PER « porteur de dynamique » a réalisé ses objectifs d'action, a créé une dynamique qui vient renforcer d'autres projets assurant sa pérennité. C'est le cas où la labellisation PER a pleinement joué son rôle de levier de développement, comme pour le PER «Valorisation de la forêt par le développement du bois-énergie et du frêt ferroviaire » du PNR Livradois-Forez, qui a su impulser une dynamique de développement intégrée autour des ressources forestières et de la voie ferrée (Lardon et al., 2012).

\section{...̀̀ la mise en œuvre de la gouvernance territoriale}

Dans l'approche par les trajectoires de développement, sur le corpus des 29 PER du Sud-ouest, nous avons reconstruit l'historique de l'émergence des PER. Nous avons étudié comment les PER s'articulaient avec des dispositifs de développement déjà existants, notamment des programmes Leader ou des politiques territoriales de type Pays, en vue de comprendre comment le PER s'insérait dans ces trajectoires et s'il jouait à cet égard un rôle positif (rôle d'activateur ou d'accélérateur) ou négatif (rôle perturbateur). Nous avons essayé enfin d'identifier les éventuels effets positifs sur la gouvernance territoriale à travers une grille de lecture des innovations (Fontan et al., 2004) apportées par le PER. Un premier aspect de cette grille, dit méthodologique, s'intéressait aux liens entre acteurs et à l'amélioration de la coordination entre eux, par exemple en étudiant si la démarche PER permettait d'apaiser des conflits ou bien de susciter la création de liens nouveaux entre des acteurs différents avec la formulation d'un intérêt commun (une complémentarité économique par exemple). C'est essentiellement cet aspect qui concernait la gouvernance territoriale. Le second aspect regardait les innovations techniques, c'est-à-dire les apports contribuant à la structuration d'une filière (équipements de mise à niveau), à 
l'amélioration de pratiques professionnelles (apprentissages, formation) ou encore à l'élargissement des débouchés commerciaux. Un aspect d'innovation institutionnelle pouvait apparaître enfin, dans les quelques cas où le PER a permis, ou accompagné, une recomposition plus complète du système d'acteurs et l'émergence d'un territoire de projet.

Cet objectif a amené à croiser la dynamique de réalisation du PER et ce qui s'opérait en matière de gouvernance territoriale (figure 4). On constate que le PER est un dispositif qui n'agit pas nécessairement dans le sens d'une amélioration et d'un enrichissement de la gouvernance.

Pour la catégorie 1, le PER a atteint ses objectifs opérationnels (compris ici en termes de réalisation, sans appréciation sur la performance des actions réalisées) et il produit des effets positifs en termes de gouvernance territoriale. Dans la catégorie 3, le PER n'a pas pu concrétiser les principales actions projetées mais il a joué en revanche un effet bénéfique en matière de gouvernance. Pour ces deux situations, dans la plupart des cas, les bénéfices en matière de gouvernance ont d'abord résidé dans la construction d'une expertise commune sur les enjeux de développement. La préparation d'une candidature a généralement stimulé des liens déjà existants entre des acteurs du territoire (socioprofessionnels d'un même secteur économique, associations en lien avec des collectivités par exemple) mais qui ne s'étaient pas forcément concrétisés en termes de coordination structurée. Elle a également pu initier de nouveaux rapprochements d'acteurs, voire la construction de partenariats plus étroits, parfois formalisés dans le cadre de conventions. En incitant à présenter des «projets innovants » (Alvergne et De Roo, 2008), la démarche a également pu amener certains acteurs, jusque-là non agissants ou désintéressés par les enjeux de développement territorial, à s'y impliquer (effet d'activation ou de consolidation de ressources territoriales). C'est enfin à la construction d'une capacité d'autonomie de décision du système local d'acteurs (empowerment, capacité à réagir et à anticiper) qu'ont contribué certains PER.

Pour la catégorie 2, le PER a atteint globalement ses objectifs de réalisation, sans toutefois rien apporter de neuf à la gouvernance territoriale. C'est généralement le cas de projets de PER très opérationnels, structurés autour d'une action phare et contrôlés par une structure porteuse qui est le leader du projet. Le dispositif n'a pas constitué une scène de projet territorial. Enfin, pour la catégorie 4, le PER a complètement échoué ou alors n'a pas bien fonctionné, même si la réalisation de quelques opérations a parfois pu être assurée. Ce résultat globalement décevant est en partie lié au fait que des problèmes majeurs de gouvernance sont survenus
Figure 4 : Typologie de la gouvernance territoriale et de la réalisation des actions par l'approche des trajectoires de développement (d'après Barthe et al., 2009)

A typology of territorial governance and achievements using trajectories development methodology

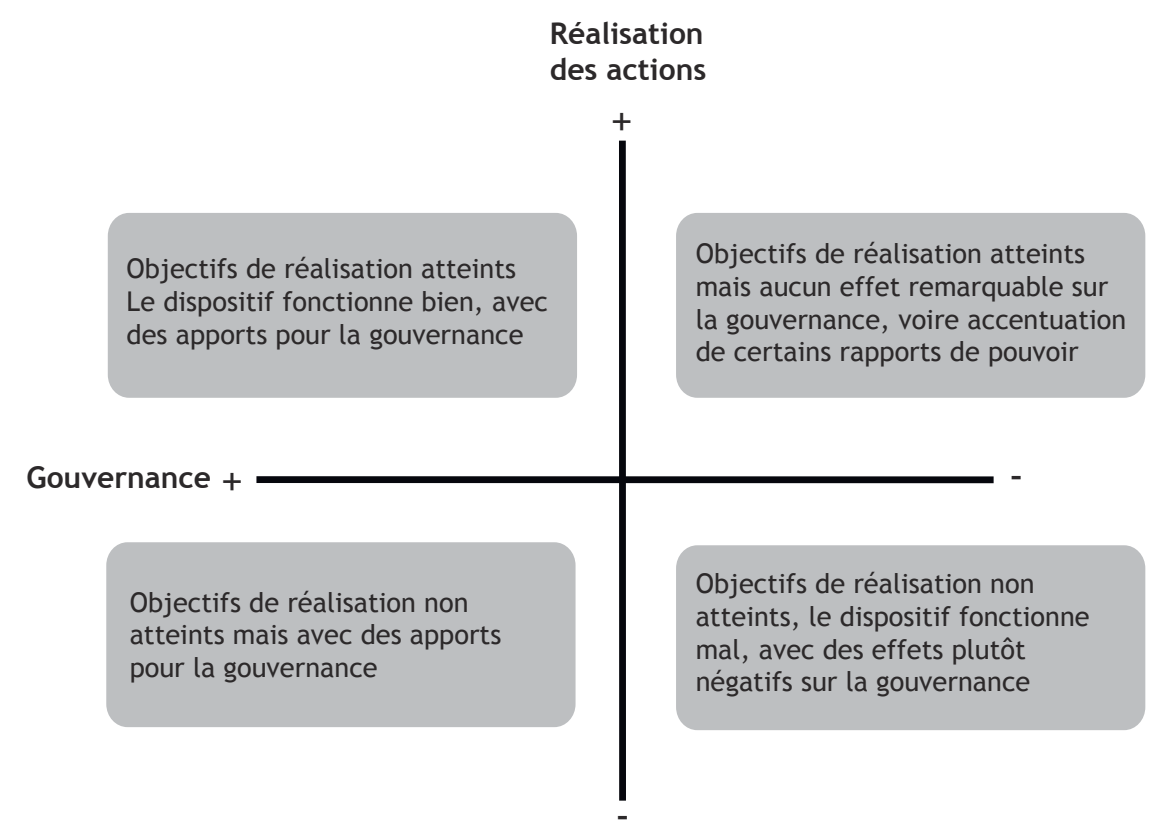


(absence d'animateur clairement défini par exemple) et n'ont pas pu être surmontés. D'autres projets ont connu des difficultés majeures car n'ayant pas tenu compte des orientations déjà définies par le projet de territoire ou étant demeuré sans lien fonctionnel réel avec des structures institutionnelles et professionnelles (projet « hors sol »).

\section{REgARDS CROISÉS}

La comparaison du classement des 6 PER communs aux deux approches dans les typologies respectives (configuration socio-spatiale et trajectoire de développement) met en évidence une convergence du potentiel de développement des territoires et des modalités de gouvernance. Lorsque les conditions de mise en œuvre effective du projet sont réalisées, les résultats convergent entre l'analyse menée à partir d'enquêtes directes sur la gouvernance et la réalisation des actions (analyse des trajectoires de développement) et celle conduite sur les projets énoncés dans les dossiers et confrontés aux réalités de terrain (analyse des configurations socio-spatiales). La cohérence du projet dans sa continuité semble être garante d'une réelle gouvernance territoriale. Des écarts apparaissent cependant lorsque le projet PER ne correspond pas, dans la réalisation de ses actions, à ce qui était initialement énoncé dans le dossier, mais correspond cependant aux objectifs des porteurs de projets enquêtés. Il s'agit là à la fois de différence de méthode d'approche (l'enquête par panel d'entretiens s'avère plus précise que l'analyse des dossiers), mais aussi d'appréciation sur «ce qui fait développement pour le territoire » (un bilan limité à la réalisation d'une seule action ou d'une étude de faisabilité ne suffisent pas à qualifier le PER de « réussi » in fine). Un autre écart d'appréciation entre les analyses existe lorsque la dynamique enclenchée sur le terrain ne semble pas aussi ambitieuse qu'elle aurait pu l'être, pour un opérateur ayant des capacités fortes en matière d'ingénierie et d'animation territoriale, comme un PER porté par un PNR par exemple.

L'ensemble des PER étudiés par nos deux équipes montre que l'on peut bien interpréter les projets tels qu'énoncés dans les dossiers en termes de capacité d'organisation des acteurs et de potentiel de développement des territoires. Réciproquement, les modalités de gouvernance et les conditions de réali- sation des actions confirment l'importance des relations socio-spatiales que les acteurs ont su créer et de l'historique de constitution du projet. Les grilles d'analyse des configurations socio-spatiales et des trajectoires de développement constituent un cadre théorique commun qui met en avant les modalités de construction des territoires et les modes d'organisation collective. Elles dépassent le cas des pôles d'excellence rurale et permettent de s'interroger sur l'appropriation des dispositifs par les acteurs de terrain et de questionner en retour la conception et la mise en œuvre des politiques publiques territoriales (Barthe, 2009).

\section{Un retour d'expérience mitigé sur la gouvernance territoriale}

Les résultats produits par ces deux recherches mettent en évidence que les effets des PER sur la gouvernance territoriale sont globalement modestes. Les PER ont eu des difficultés à constituer dans la durée des véritables lieux de débat territorial. D’une part, la gestation de certains PER s'est déroulée en dehors de cette préoccupation. D'autre part, les entretiens ont montré que là où elle a tenu une place structurante, la gouvernance s'est essentiellement exprimée au lancement du dispositif, c'est-àdire au moment de la construction des candidatures. De plus, les comités de pilotage n'ont pas tenu un rôle d'animateur territorial, à la différence des programmes LEADER (Chevalier, 2014) où le comité de programmation notamment fait exister ce débat tout au long du projet. Dans le cas des PER, les échanges entre les catégories d'acteurs impliqués (services de l'État, cofinanceurs, collectivités, porteurs de projet) ont souvent glissé vers une phase de gestion technique centrée sur l'aboutissement des projets.

Deux éléments pondèrent cependant ces résultats mitigés. Tout d'abord, les conditions données par l'appel à projets ne laissaient guère de latitude (dossier de candidature à proposer sous 3 mois) pour impulser des projets nouveaux, engager des mobilisations nouvelles et élargies, bien que certains leaders et porteurs de projet soient parvenus à tenir cet objectif. Ensuite, les entretiens ont souligné combien, d'un PER à l'autre, les porteurs de projet n'avaient pas les mêmes postures dans la mobilisation de ce dispositif. Si certains PER peuvent sem- 
bler modestes dans leurs ambitions, c'est en partie parce qu'ils ont servi de relais pour l'achèvement d'une opération ou d'une réalisation engagée avec un autre dispositif (un contrat Leader par exemple).

Nous avons néanmoins observé quelques cas de renforcement de dynamiques de gouvernance territoriale (Lardon et al., 2014). Certains territoires ont utilisé le dispositif PER pour impulser une dynamique d'action collective qui se poursuit au-delà du financement direct des opérations (rôle intégrateur). Le simple aboutissement d'une candidature a pu en soi constituer un résultat, dans le cas de démarches réellement partenariales révélant une capacité des acteurs territoriaux à se fédérer et se coordonner pour porter un projet commun (rôle activateur). Pour certaines petites intercommunalités, la reconnaissance d'un PER a parfois constitué un premier acte de la légitimité de cet échelon administratif à porter un projet de développement propre pour son territoire (rôle promoteur). La mise en place du dispositif a même servi de support à des formes d'innovation institutionnelle locale, en incitant des recompositions profondes des débats sur le territoire et des modalités d'organisation dont il se dote, comme l'émergence d'un projet de PNR (rôle générateur).

Cependant, ces résultats ont révélé des postures différentes de gouvernance territoriale au sein du triptyque d'encadrement des dynamiques de développement : acteurs institutionnels (État, régions, départements)/structures intermédiaires (consulaires et organisations socioprofessionnelles/territoires locaux organisés). Ils ont tout d'abord souligné la montée en puissance des communautés de communes sur les questions de développement. Certaines ont profité de cette occasion pour réaliser leur premier projet en la matière. Les départements ont tenu un rôle clef dans le système de financement et le portage politique de nombreuses candidatures. Acteur incontournable de l'appui aux porteurs de projets, ils ont parfois «écrasé » le dispositif, soit en en prenant le leadership, soit en imposant ou conditionnant leur appui à certaines opérations. Les Pays apparaissent comme les principaux porteurs de candidature, mais ils ont fréquemment été les relais d'initiatives construites à une échelle inférieure. Ils ont rarement été leaders sur cette première génération de PER, car ne disposant que d'une capacité organisationnelle et technique limitée. Les PNR affichent des postures contrastées, nombreux étant ceux ayant mobilisé le dispositif dans une « stratégie d'aubaine » et d'opportunité pour compléter ou relayer des plans de financements d'actions déjà engagées. Certains ont cependant réussi à mobiliser le PER comme un levier de développement (Lardon et al., 2012).

Finalement, nous avons montré que la procédure PER a parfois fonctionné comme un levier de développement pour des territoires ruraux. Deux points de vigilance sont à noter pour la gouvernance territoriale. Le premier est la nécessité de favoriser les lieux et les moments de dialogue entre les différents acteurs impliqués, en vue d'une co-construction des démarches de développement. En effet, l'acteur institutionnel doit venir conforter des initiatives locales et non pas les imposer. Pour ce faire, les acteurs institutionnels tels que les PNR et les départements ont une marge de manœuvre limitée, car ils restent trop souvent sur leur posture institutionnelle, qui leur donne légitimité, mais sans beaucoup de souplesse. A contrario, d'autres structures, telles que les pays et les communautés de communes, ont des atouts pour être au plus près des acteurs locaux, mais restent trop souvent pris dans des rapports politiques, plus sectoriels que transversaux. Ainsi, ce n'est pas tant le type de structure, que la capacité de dialogue entre les acteurs, qui est nécessaire pour conforter le développement territorial. Le second point est de s'assurer l'appui d'acteurs médiateurs, qui n'étaient pas obligatoirement prévus au départ, mais que nous avons vu émerger au cours du processus. C'est ainsi par exemple que l'implication des lycées agricoles et de la recherche dans les PER Bioressources s'est révélée très profitable, pour relier les différents acteurs concernés et compenser le fait qu'il n'y avait pas d'animation prévue dans la procédure institutionnelle. Les chambres consulaires peuvent aussi aider à dépasser les contingences du local et à relier les acteurs, tout comme les associations, ce qui était peu le cas lors du démarrage des PER, mais semble maintenant possible, vu les évolutions organisationnelles en cours.

\section{Quels LEVIERS DE DÉVELOPPEMENT POUR LES TERRITOIRES RURAUX?}

Les analyses menées sur la gouvernance territoriale des PER ont mis en évidence les atouts et les limites de la procédure. De ces expériences, nous 
pouvons tirer quelques enseignements sur les modalités d'accompagnement des acteurs par une nouvelle ingénierie territoriale et soumettre quelques propositions pour élaborer une réelle politique de développement des territoires ruraux.

\section{Vers une ingénierie de la gouvernance territoriale}

Les contraintes de réalisation ont marqué le déroulement de cette première génération de PER. Elles sont dues en partie à la rigidité des engagements, mais aussi à une appréciation insuffisante de la faisabilité des projets et des moyens nécessaires, notamment en matière d'ingénierie, pour l'accomplissement des actions. Le dispositif PER a montré le besoin du renforcement de l'ingénierie territoriale : les porteurs de projet ont besoin d'accompagnement, de temps et des savoir-faire se révèlent souvent nécessaires pour faire travailler des acteurs venant d'horizons variés, comme pour expliciter des démarches (Piveteau, 2010). Or le contexte rural à cet égard se singularise souvent par des situations très contrastées en matière de compétences disponibles. Nombre de territoires ruraux ne disposent ainsi que d'une ingénierie incomplète, souvent moins structurée que dans les territoires urbains, où notamment les services de l'État comme des collectivités sont plus présents. Cette situation s'est dégradée depuis le lancement de la réforme des collectivités en 2010, avec l'affaiblissement d'un certain nombre de structures animatrices du développement comme les pays.

Hélène Rey-Valette et al. (2011), dans un guide pour la mise en œuvre de la gouvernance en appui au développement durable des territoires, proposent de constituer un outil qui concourt à renforcer l'ingénierie de la gouvernance territoriale. Celle-ci est définie comme "l'ensemble des méthodes et outils permettant la coordination, la participation, la collaboration et l'apprentissage des acteurs au pilotage des projets de territoires ». Les points clés retenus en sont : le partage de connaissances sur la question de la gouvernance territoriale et de formes de partenariat complémentaires, la nécessité, cheminfaisant, de faire évoluer les échelles d'observation, et les partenariats, à la fois du fait du caractère multi niveaux des processus et en raison de la complexité des contextes. Ils permettent de rendre compte et de comparer la diversité des formes possibles de mise en œuvre de cette gouvernance territoriale. L'opérationnalisation de la gouvernance territoriale repose sur cinq propriétés : la participation, l'organisation du pilotage, la transversalité, l'évaluation et l'amélioration continue. Participation, organisation du pilotage et évaluation positionnent le cadre des dispositifs et actions menées. La transversalité et l'amélioration continue sont des objectifs plus généraux qui posent des questions pour intégrer ces principes dans les procédures. Elles conduisent à des démarches adaptatives impliquant la prise en compte du long terme et nécessitant des processus d'apprentissage collectif dans une perspective de développement durable (Rey-Valette et al., 2008).

La chaîne d'ingénierie territoriale (Lardon, 2011), définie comme «la façon dont les acteurs s'organisent, tout au long de la conduite du projet, selon différentes temporalités et modalités » permet de répondre à ces cinq propriétés. Il s'agit de comprendre quels sont les référentiels institutionnels mobilisés par les acteurs et comment de nouveaux acteurs émergent, dans le déroulement d'un projet de territoire, afin de développer de nouvelles compétences. Il s'agit également d'accompagner ces dynamiques de changement, par l'apport de modèles théoriques et par leur mise à l'épreuve sur le terrain. Cela amène à construire des modèles et outils pour rendre compte des transformations, dans le temps et dans l'espace, des coordinations d'acteurs, des combinaisons d'actions et des compositions d'espaces. La mise en œuvre de tels principes d'ingénierie est en cours de construction dans les territoires.

\section{Pour une politique de développement des territoires ruraux}

Inscrire les PER dans une politique de développement rural plus transversale et mieux structurée sur le long terme (Datar, 2003), à l'instar de ce qui se fait dans d'autres pays (Gouvernement du Québec, 2006; 2013; Le Blanc et al., 2007) pourrait avoir un effet déterminant sur les dynamiques de développement des territoires.

Nous pouvons faire quelques recommandations relatives à l'accompagnement que la DATAR aurait pu mener auprès des territoires de projet, afin de capitaliser ces expériences. La DATAR aurait pu jouer un rôle fédérateur, car les territoires et les 
porteurs de projet en étaient demandeurs. Plusieurs propositions en direction des acteurs institutionnels peuvent ainsi être tirées de nos analyses. Elles ouvrent également des pistes sur les potentiels de développement et d'innovation des acteurs ruraux. Ces potentiels sont mis en lumière tant dans l'évolution des lieux et des formes de l'action territoriale, que dans la transformation de l'espace de formation que nécessite la professionnalisation des acteurs pour rendre reproductibles les compétences valorisées.

La première proposition consiste à reconnaître la diversité des formes d'organisation des territoires (échelles, portage, combinaisons d'acteurs, objectifs de réalisation, etc.) et d'évaluer les projets en prenant en compte leurs natures différentes. Le croisement de nos analyses sur les PER a bien montré l'existence d'une diversité de leviers de développement possibles, en écho à des postures et des objectifs différents formulés par les acteurs territoriaux en fonction de situations multiples quant aux formes d'organisation de l'action collective, de régulation, de conduite de projet, etc. Ces leviers ne sont pas partout adaptables, interchangeables ni pertinents. Il faut donc de la souplesse dans leur emploi, que l'institution devrait reconnaitre et favoriser. Les critères d'évaluation doivent être multiples, mais pour cela, il est nécessaire de mieux connaitre les projets. L'analyse des configurations socio-spatiales est un outil qui pourrait être facilement utilisable à ces fins.

La seconde proposition est de faciliter l'articulation entre plusieurs types d'acteurs et plusieurs types d'actions. La condition pour qu'un projet de territoire soit un levier de développement, c'est qu'il y ait un lien entre la dimension institutionnelle apportée par la politique publique et la capacité d'innovation des acteurs locaux. Cela passe par les réseaux de dialogue entre les acteurs concernés et par des dispositifs de concertation, pour construire ensemble un projet de territoire. L'incitation à l'innovation et l'institutionnalisation de ces instances de dialogue, telles que définies dans l'approche des trajectoires de développement, pourraient servir de modèle pour une meilleure gouvernance territoriale.

La troisième proposition est d'aider à gérer l'incertitude dans la dynamique de transformation des territoires, donc dans les métiers des acteurs du développement territorial. Cela pose la question des compétences et de la formation de ces acteurs. De nouvelles compétences sont à inventer pour accompagner le développement territorial. La formation est un laboratoire d'expérimentation de ces nouvelles formes d'intervention sur le terrain, qui rend reproductible non pas les solutions mais la façon de résoudre les problèmes, en situation (David et al., 2001). L'accompagnement de l'ingénierie territoriale par la formation constitue vraisemblablement une piste d'avenir pour conforter les projets et coordonner les dynamiques de développement territorial.

\section{Conclusion - Perspectives de recherche}

Les analyses produites sur la première génération de PER permettent de suivre les évolutions récentes, tant dans les conceptions que dans les pratiques, de l'action de développement dans les territoires ruraux. Au-delà de ces apports, ces travaux renouvellent aussi les questions de recherche sur les formes et la conduite de ce développement par les expérimentations et questions qu'ils soulèvent. Les expérimentations diverses ainsi observées dans les PER pourraient être transférées vers d'autres territoires et promouvoir des manières originales de penser et de faire le développement territorial.

Aussi diversifiés soient-ils dans leurs configurations propres (thématiques investies, systèmes d'acteurs, contexte politique, etc.), les PER n'épuisent pas les situations et les problématiques soulevées par les enjeux du développement territorial. Les travaux de la recherche évaluative ont d'ailleurs montré les limites d'un dispositif finalement conçu de manière très détachée des autres instruments d'appui au développement rural, notamment des politiques territoriales menées par les collectivités. Les approches proposées interrogent « la capacité des territoires à savoir s'organiser » selon la formule de P. Dartout (ex-délégué interministériel DATAR), mais également le sens donné aux choix des acteurs et aux outils mis à leur disposition. À travers les PER, nous nous interrogeons aussi sur les évolutions des conceptions de la ruralité et des réponses proposées par la puissance publique pour construire ou consolider les leviers de développement.

Analyser les formes de développement territorial, les comparer et les mettre à l'épreuve sont des perspectives très stimulantes. Les analyses que nous avons menées sur les pôles d'excellence rurale nous 
semblent dépasser ce cas spécifique et s'adapter à tout projet de territoire, qu'il soit rural, urbain ou péri-urbain, articulant des initiatives locales et institutionnelles. L'ensemble des travaux sur les territoires de projet peut ainsi en être enrichi. Pour aller plus loin, on peut aussi penser que cette approche peut être utile à différentes échelles, pour comprendre comment chaque territoire s'organise et comment ils contribuent à une cohérence globale. La nouvelle politique d'égalité des territoires (AdCF, 2014) pourrait trouver là concepts, méthodes et outils pour le développement territorial.

\section{Bibliographie}

AdCF, 2014. Loi de modernisation de l'action publique territoriale et d'affirmation des métropoles (MAPAM). Note juridique, 18 p. [http://www.adcf.org/files/AdCF_note_ MAPAM_jan2014.pdf].

Alvergne Ch., De Roo P., 2008. Les pôles d'excellence rurale : regards sur une politique française en direction de l'espace rural, Organisations et Territoires, Vol. 17, $\mathrm{n}^{\circ} 1$.

Angeon V., Caron P., Lardon S., 2006. Des liens sociaux à la construction d'un développement territorial durable. Quel rôle de la proximité dans ce processus?, Revue Développement durable et territoires (http://developpementdurable. revues.org/).

BARThe L., 2009. Le développement territorial dans les espaces ruraux, nouveau défi des politiques publiques, in Compagnone C., Auricoste C., Lemery B. (dir.). Conseil et développement en agriculture: quelles nowvelles pratiques?, Dijon/Versailles, Educagri/Editions Quae Cemagref/Cirad/ Ifremer/Inra, coll. « Sciences en Partage ».

Barthe L., Milian J., Taulelle F. (dir.), 2009. Les pôles d'excellence rurale : processus, gouvernance et plus-value dans les trajectoires de développement des territoires. DIACT, 164 p. [http://www.datar.gouv.fr/sites/default/files/datar/2009-perprocessus-gouvernance-plus-value-toulouse2.pdf].

Berger A., Chevalier P., Cortès G., M. Dedeire, 2009. Héritages et trajectoires rurales en Europe, Paris, L'Harmattan, coll. « Logiques sociales », 253 p.

Chevalier P., 2014. Action locale et développement rural en Europe - Le modèle européen LEADER 2007-2013, intégration and social cohésion (coll.), RISC, $\mathrm{n}^{\circ} 14$, Peter Lang Ed., Bruxelles, 202 p.

DATAR, 2003. Quelle France rurale pour 2020? Contribution à une nouvelle politique de développement rural durable, La Documentation française, Paris, $64 \mathrm{p}$.

DAvid A., 2001. La recherche-intervention, généralisation des méthodes de recherche en management?, in David A., НATCHUEL. A, LAUFER C, Les nowvelles fondations des sciences de gestion, Vuibert, mars 2001 [seconde édition : 2008; troisième édition : 2012].
Deffontaines J.-P., Marcelpoil E., Moquay P., 2001. Le développement territorial : une diversité d'interprétations, in Lardon S., Maurel P., Piveteau V. (éds.), Représentations spatiales et développement territorial. Bilan d'expériences et perspectives méthodologiques, Paris, Hermès, p. 39-56.

Fontan J-M., Klein J-L., Tremblay D-G., 2004. Innovation et société : pour élargir l'analyse des effets territoriaux de l'innovation, Géographie, économie, société, Vol. 6, n² 2 , p. $115-128$.

Gilly J.-P., Leroux I., Wallet F., 2004. Gouvernance et proximité, In Pecqueur B., Zimmerman J.-B., Économie de proximités. Hermès Science Publications, $264 \mathrm{p}$.

Gouvernement du Québec, 2006. Politique nationale de la ruralité, 2007-2014: une force pour tout le Québec, 68 p. [http://www.mamrot.gouv.qc.ca/pub/developpement_regional/ ruralite/politique_nationale_ruralite/ruralite_politique.pdf].

Gouvernement du Québec, 2013. Politique nationale de la ruralité, 2014-2024: une approche intersectorielle pour agir ensemble au sein de la MRC, 58 p. [http://www.mamrot.gouv. qc.ca/pub/developpement_regional/ruralite/politique_nationale_ruralite/PNR_2014-2024.pdf].

Guigou J.-L., 2008. Douze convictions pour une nouvelle pensée territoriale, In JeAn Y., Vanier M., La France, aménager les territoires, Paris, Armand Colin, coll. «U », p. 6-18.

Gumuchian H., Pecqueur B., (dir.) 2007. La ressource territoriale, Paris, Economica, coll. «Anthropos », 252 p.

IDER F., 2010. Evaluation et analyse du potentiel de développement des pôles d'excellence rurale. Application de la grille des configurations socio-spatiales à un échantillon de PER. Mémoire de recherche master DTNR M2 TAM, Université B. Pascal, Clermont-Ferrand, 129 p.

Jean Y., Vanier M., La France, aménager les territoires, Paris, Armand Colin, Collection U, 2008, 335 p.

Lardon S., Piveteau V., 2005. Méthodologie de diagnostic pour le projet de territoire : une approche par les modèles spatiaux, Géocarrefour, Vol. 80, n² 2, p. 75-90.

Lardon S., Chia E., Rey-Valette H. (dir.), 2008. Outils et dispositifs de la gouvernance territoriale, Norois, $n^{\circ} 209,4$, $177 \mathrm{p}$.

Lardon S., Cayre P. (dir.), 2009. Pôles d'excellence rurale: de nouveaux modèles de développement pour les territoires ruraux?, DIACT, 43 p. [http://www.datar.gowv.fr/sites/default/ files/datar/2009-per-modeles-de-developpement-engref.pdf].

LARDON S., 2011. Chaîne d'ingénierie territoriale : diversité des acteurs dans la conduite d'un projet de territoire, in DAYAN L., Joyal A., Lardon S. (dir.), L'ingénierie de territoire à l'épreuve du développement durable, Paris, L'Harmattan, $284 \mathrm{p}$.

Lardon S., Loudiy S., Cournut E., Fournier J., 2012. Les configurations socio-spatiales : outil et dispositif de gouvernance des territoires, Revue d'Auvergne, $\mathrm{n}^{\circ}$ 602-603, p. 295-310.

Lardon S., Barthe L., Ider F., Loudiyi S., Milian J., Taulelle F., 2014. La labellisation des Pôles d'excellence rurale. Vers de nouveaux modèles de développement pour les territoires ruraux?, in Fournier M. (dir.), Labellisation et 
mise en marque des territoires, CERAMAC n ${ }^{\circ} 34$, ClermontFerrand, Presses Universitaires Blaise Pascal, p. 325-342.

LeBlanc P. (dir.), Jean B., Lafontaine D., Marceau R., Belley S., Boisjoly J., Chiasson G., Joyal A., Plante S., RobiTAILlE M., 2007. Recherche évaluative sur le dispositif des pactes ruraux et ses retombées. Rapport de recherche 1: Le modèle et les indicateurs de suivi et d'évaluation du dispositif $d u$ pacte rural, CRDT-UQAT et CREXE-ÉNAP.

Leroux I., 2006. Gouvernance territoriale et jeux de négociation. Pour une grille d'analyse fondée sur le paradigme stratégique, Négociations, n ${ }^{2}$, p. 83-98.

Mainet H. (dir.), 2009. La mobilisation des ressources pour des projets d'excellence dans des territoires fragiles, Rapport pour la DIACT, 123 p. [http://www.datar.gouv.fr/sites/default/files/ datar/2009-per-mobilisation-des-ressources-ceramac.pdf].

Milian J., Bacconnier S., 2014. Requalifier les territoires de l'action locale : l'exemple des pôles d'excellence rurale de la filière bois, Territoires en Mouvement, $\mathrm{n}^{\circ}$ 22, p. 54-66.

Pasquier R., Simoulin V., Weinsbein J., 2007. La gouvernance territoriale-Pratiques, discours et théories, Droit et Société, $235 \mathrm{p}$.

Pecqueur B. (coord.), 2009. La place du patrimoine et de la culture dans les projets de développement territorial, Rapport pour la DIACT, 63 p. [http://www.datar.gouv.fr/sites/default/ files/datar/2009-per-place-du-pratimoine-cermosem.pdf].
Perraud R., 2009. Les configurations socio-spatiales et temporelles des Pôles d'Excellence Rurale : itinéraire méthodologique et construction d'archétypes à partir des 21 PER d'Auvergne. Mémoire de Master «Développement des territoires et nouvelles ruralités », Clermont-Ferrand, université BlaisePascal, 88 p. + annexes.

Piveteau V., 2010. L'ingénierie territoriale : pour un parti pris géographique, Mémoire de HdR en Géographie, ENS Lyon, $114 \mathrm{p}$.

Rey-Valette H., Lardon S., Chia E. (dir.), 2008. Governance: Institutional and learning plans facilitating the appropriation of sustainable development, International Journal of Sustainable Development, Volume 11, n²/3/4, p. 101-287.

Rey-Valette H. (dir.), 2011. Guide pour la mise en œuvre de la gouvernance en appui au développement durable des territoires (C) Cemagref, CNRS, Geyser, Inra, Supagro, Université Montpellier 1. Diffusion INRA-Montpellier, 155 p.

TAulelle F., 2008. Une lecture de la politique d'aménagement du territoire à travers les zonages et les pôles. In JeAn Y., Vanier M., La France, aménager les territoires, Paris, Armand Colin, coll. «U», p. 242-256

Woessner R., 2010. La territorialisation : proposition pour la compréhension du phénomène par une entrée systémique. Revue d'Économie Régionale et Urbaine, 2010/4, p. 669-685. 